\title{
Physicians, Hippocrates and biosimilars: applying ancient principles in a modern society
}

Adjunct Professor Pekka Kurki, MD, PhD

Physicians are pondering the clinical use of biosimilars. A reliance on clinical trials is deeply rooted in the modern healthcare system, whereas comparability and totality of evidence remain unknown concepts. This editorial explores these ideas, with reference to a case study of Italian gastroenterologists.

Keywords: Biosimilars, NOR-SWITCH, totality of evidence

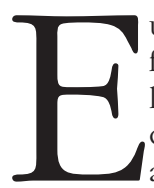
urope's healthcare systems are facing ever-increasing costs. Biologicals, a group of biotechnologyderived therapeutic products, are driving the increasing costs of pharmacotherapy. Unless these increasing costs can be contained, the use of new biological medicinal products could be restricted, even in the wealthiest European Union (EU) Member States.

One means of avoiding such price hikes is developing drugs that are equivalent to brand-name products, but without the patent. Copies of biological products are known as biosimilars. In the EU, cheaper copies of some of the best-selling 'blockbuster' biologicals, such as etanercept, infliximab (tumour necrosis factor-alpha $[\mathrm{TNF}-\alpha]$ inhibitors used to treat autoimmune diseases such as arthritis) and insulin glargine (a long-acting insulin analogue) have been licensed. Biosimilars to other blockbuster biologicals are in the evaluation or development phases [1]. In the EU, biosimilars are developed according to stringent regulatory requirements and those licensed have been shown to be safe and efficacious after over a decade of use.

In principle, biosimilars have the potential to reduce costs and increase access for important medicines. However, the adoption of biosimilars has faced barriers within the healthcare system. With the exception of infliximab, most of the blockbuster biological drugs are used outside of hospitals, within the community. Thus, physicians are in a key position for increasing the use of biosimilars. Illustrating this point, the paper recently prepared by Annese et al. [2] describes use of biosimilars in the Italian healthcare system, including the difficulties doctors face in accepting biosimilars which often bring economic relief but no direct clinical improvements over existing drugs.

The authors also discuss the Italian health insurance system, which provides pharmacotherapy for citizens. In this system, neither physicians nor patients have an incentive to prescribe and use biosimilars. The system is also fragmented and the use of biosimilars varies between regions. Such variation is difficult to explain by medical or scientific reasons. Together, the lack of incentives and lack of coordination between different regions hinder the rational use of biosimilars. Sadly, this situation is not unique to Italy - healthcare systems across the EU have been poorly prepared for the entry of biosimilars.

As in most EU Member States, the substitution of biosimilars at the pharmacy level is not permitted in Italy. Thus, the decision to initiate treatment with a biosimilar or to switch treatment from the originator drug (reference product) to a biosimilar is normally made by hospitals or individual physicians and patients. The Italian Medicines Agency (Agenzia Italiana del Farmaco, AIFA) states in its recent concept paper that biosimilars are safe and efficacious and that a switch from a reference product to its biosimilar copy is possible, but leaves the responsibility for the decision to individual physicians [3]. In contrast, local medical societies, such as Italian Society of Rheumatology, Italian Society of Dermatology, and Italian Group of Inflammatory Bowel Disease, are less positive regarding the use of biosimilars, especially when it comes to blockbuster monoclonals, such as those used to treat autoimmune diseases $[4,5]$. Thus, on the whole, Italian physicians, like many of their colleagues in other EU Member States, appear to distrust licensed biosimilars.

The paper of Annese et al. illustrates the dilemma from the point of view of gastroenterologists. On the one hand, it is acknowledged that biosimilars may enable cost savings, improve access to treatment and promote sustainable health care. On the other hand, local medical societies and individual physicians are reluctant to recommend the use of biosimilars.

The willingness of Italian gastroenterologists to accept the biosimilar concept was tested after the launch of the first biosimilar monoclonal antibody, infliximab, in Italy. The sore points for clinicians were the extrapolation of safety and efficacy from another therapeutic indication (rheumatoid arthritis) to inflammatory bowel disease (IBD) and the interchangeability of biosimilars and their reference products. Italian gastroenterologists insist

Author: Adjunct Professor Pekka Kurki, MD, PhD, University of Helsinki, 19 Lukupolku, Fl-00680 Helsinki, Finland

Submitted: 24 October 2016; Revised: 1 November 2016; Accepted: 1 November 2016; Published online first: 7 November 2016 
that clinical trials must be performed in IBD, even if comparability has been demonstrated on physicochemical, structural, functional, pharmacokinetic, safety and efficacy levels in rheumatoid arthritis.

However, there is good evidence for the interchangeability of biosimilars. Several small, mainly uncontrolled studies have not raised concerns. The preliminary results of a large controlled Norwegian switch study (NOR-SWITCH) of biosimilar infliximab in its major therapeutic indications, including IBD, indicate no significant differences in safety and efficacy to the reference product [6]. These findings, along with the good safety record of EU biosimilars, suggest that biosimilar and reference products can be switched safely.

These results may not satisfy all gastroenterologists. However, it is extremely difficult to detect small differences in drug activity due to the fluctuation in disease course and pharmacokinetics within an individual patient, as well as variation between production batches of biological products. A demonstration of the lack of such minor differences would require very large studies and make biosimilar development unfeasible. Thus, one has to rely on comparability based on the totality of evidence from physicochemical, structural and functional tests as well as from limited clinical studies. Furthermore, Italian gastroenterologists are reluctant to switch a patient to a biosimilar if their disease is being well controlled with the reference product. This is another blow to biosimilars, because a switch to biosimilar infliximab only makes clinical sense in patients who already respond to infliximab therapy.

According to Annese et al., the patient should be informed that the safety profile of a biosimilar is less well known than that of the reference product. They also refer to legislation that requires physicians to choose the safest alternative product. Taking this advice literally would limit prescriptions of not only biosimilars but also generic (small molecule) drugs.

European regulators maintain the position that biosimilars contain a new version of the active substance of its reference product, just as a manufacturing change will create a new version of a biological product. Thus, the fact that the original infliximab has had more than 30 changes to its manufacturing process without any supporting clinical safety and efficacy studies is difficult to reconcile with the Italian position towards biosimilars, which are developed according to the same principles of comparability.

To support their conservative approach to biosimilars, Annese et al. refer to Hippocrates' oath cited as 'first, do not harm'. Some scholars of ethics say that Hippocrates actually said, 'above all, do not harm more than succour'. The situation becomes even more complex if one considers biosimilars at an interpersonal level. Vaccines provide an extreme example of the problem of the concept 'first, do not harm'. It has been argued that the benefits to the many outweigh the harms to the few [7]. With biosimilars, the harm would be theoretical and the benefit economical.

The current circumstances, whereby the physician is part of the healthcare system and where responsibility for the sustainability of health care, including the costs of pharmacotherapy, lie on administrative and political levels, which is very far from the circumstances that prevailed during Hippocrates' time. For biosimilars, prescribers need to balance the theoretical harm to patients with the concrete benefit of improving access to medicines. What would Hippocrates' advice be today - to do nothing or act?

Annese et al. insist that the choice of the therapy, originator or biosimilar, should be left to the physician and the patient. Because of the slow uptake of biosimilars, this view has already been challenged and even overruled in several countries, including some regions in Italy that have issued administrative orders to prescribe biosimilars [2]. A massive and almost complete switch from the original (reference) infliximab product to its biosimilar copy took place in Denmark in 2015, following the recommendation of an official body [8]. Only 11 adverse effects related to the switch of more than $90 \%$ of patients treated with the reference product to a biosimilar infliximab were reported even after a stimulated reporting.

It seems clear that healthcare systems will gradually adopt biosimilars, including the switches from the reference products to biosimilars. The important question is: will physicians be part of the solution or part of the problem?

Competing interests: None.

Provenance and peer review: Commissioned; internally peer reviewed.

\section{References}

1. European Medicines Agency. Applications for centralised marketing authorisation [homepage on the Internet]. [cited 2016 Nov 1]. Available from: http:// www.ema.europa.eu/ema/index.jsp?curl=pages/ regulation/q_and_a/q_and_a_detail_000169. jsp\&mid=WC0b01ac0580a45420

2. Annese V, Gabbani T, Annese AL. Biosimilars in Italy: a gastroenterologist's view Generics and Biosimilars Initiative Journal (GaBI Journal). 2016; 5(3):131-3. doi: 10.5639/gabij.2016.0503.033

3. Italy's AIFA outlines challenge of sustainable innovation, proposes important pro-biosimilars change, second concept paper. IHS Markit. 2016 Jun 16.

4. Annese V, Vecchi M. Italian Group for the Study of IBD (IG-IBD). Use of biosimilars in inflammatory bowel disease: statements of the Italian Group for Inflammatory Bowel Disease. Dig Liver Dis. 2014;46(11):963-8

5. Fiorino G, Girolomoni G, Lapadula G, Orlando A, Danese S, Olivieri I, et al. The use of biosimilars in immune-mediated disease: a joint Italian Society of Rheumatology (SIR), Italian Society of Dermatology (SIDeMaST), and Italian Group of Inflammatory Bowel Disease (IG-IBD) position paper. Autoimmun Rev. 2014;13(7):751-5.

6. Moe L. Nor-Switch: - Safe switch to biosimilar. Dagens Medicin. 2016 Oct 17.

7. Sokol DK. First do no harm revisited. BMJ. 2013; 347:f6426.

8. Danish Medicines Agency. Special focus on reported adverse reactions to biological medicines and biosimilars. 2016 [homepage on the Internet]. [cited 2016 Nov 1]. Available from: https:// laegemiddelstyrelsen.dk/en/news/2016/ /media/ FF5865ABAC6D492AB650739216D65DCB.ashx DOI: 10.5639/gabij.2016.0504.039

Copyright (c) 2016 Pro Pharma Communications International 